THE KURUME MEDICAL JOURNAL Vol. 17, No. 2, 1970

\title{
ANGIOGRAPHIC EXTRAVASATION SHADOW CAUSED BY TORN MENINGEAL VESSELS IN THE COURSE OF TRAUMATIC EXTRADURAL HEMATOMA
}

\author{
SHINKEN KURAMOTO, MITSUO WATANABE, \\ AND HIROYUKI AIBA \\ Neurosurgical Clinic, Kurume University Hospital, \\ Kurume, Japan
}

(Received for publication April 30, 1970)

INTRODUCTION

Of late, for the purpose of diagnosing an intracranial hematoma at the acute stage of head injuries, cerebral angiography has come to be employed widely and its diagnostic value has gradually been estimated higher. Therefore, the report discribing the angiographic picture of extravasation of contrast medium due to torn meningeal vessels has been made by many authors. Since we have experienced 12 cases in which the extravasation shadow of traumatic extradural hematoma was demonstrated, they are reported.

\section{MATERIAL}

Out of 64 cases of traumatic extradural hematoma treated at our clinic from January 1963 to January 1970, in 62 cases cerebral angiography was performed preoperatively. Excepting 13 cases that were difficult to read in detail owing to poor filling, 51 cases of cerebral angiography were investigated. They were composed of 47 males and 4 females. The age ranged from 5 to 75 years, and 33 cases of 21 to 50 years commanded a large majority. The site of hematoma was right in 31 cases and left in 20 cases, and the skull fracture was confirmed in 49 cases. The entire 51 cases were operated on and at the time of surgery 17 cases were found associated with cerebral contusion or subdural hematoma. Thirteen cases died and the remaining 38 cases were cured.

\section{METHOD}

Cerebral angiography was performed routinely and in most cases it was made through the common carotid artery under local anesthesia. As a contrast medium Urografin of $60 \%$ was used. X-ray films were taken with regard to the frontal and lateral arterial phases and the lateral venous phases. In 3 cases a selective angiography of the external carotid artery was conducted for differentiating extradural and intradural hematomas. 


\section{RESULTS}

On 51 cerebral angiographies of traumatic extradural hematoma 12 cases (23.5\%) revealed extravasation. As shown in the Table 1, the age ranged from 15 to 75 years and all the cases were composed of males. Hematomas were found on the right side in 6 cases and on the left in 6 cases. The interval from injuries to cerebral angiography ranged from 3 hours to 8 days and it was within 27 hours in 9 cases. The fracture on the hematoma side was demonstrated in all the cases but one. Craniotomy carried out in these 12 cases disclosed that the site of hematoma was all supratentorial and most frequent in the temporal and frontal region. Hematomas weighed 20 to $200 \mathrm{~g}$. Nine of 11 cases were completely cured and 3 died.

TABLE 1

Analysis of cases

\begin{tabular}{c|c|c|c|c|c|c|l}
\hline $\begin{array}{l}\text { Case } \\
\text { No. }\end{array}$ & Age & Sex & $\begin{array}{l}\text { Interval between } \\
\text { trauma and } \\
\text { angiography }\end{array}$ & $\begin{array}{c}\text { Side of } \\
\text { the } \\
\text { hematoma }\end{array}$ & $\begin{array}{c}\text { Weight of } \\
\text { the } \\
\text { hematoma }\end{array}$ & $\begin{array}{l}\text { Pattern of } \\
\text { extravasa- } \\
\text { tion shadow }\end{array}$ & \multicolumn{1}{|c}{ Outcome } \\
\hline 1 & 38 & M. & 96 & Right & 70 & I & Survival \\
2 & 45 & M. & 72 & Left & 20 & I & Surviva \\
3 & 15 & M. & 7 & Left & 90 & I & Survival \\
4 & 47 & M. & 27 & Left & 100 & I & Survival \\
5 & 42 & M. & 13 & Right & 60 & I & Survival \\
6 & 53 & M. & 4 & Right & 90 & IV & Death (Pneumonia) \\
7 & 22 & M. & 192 & Left & 100 & I & Survival \\
8 & 36 & M. & 15 & Right & 100 & III (with I) & Death (Cerebral \\
herniation)
\end{tabular}

Findings of cerebral angiography were all obtained as an intracranial space taking mass. Figs. 1, 2, and 3 showed only the relation between the middle meningeal artery, the extravasation shadow and the fracture line. The extravasation shadow visualized angiographically in these 12 cases was divided into 4 patterns.

Pattern I : As seen in cases of $1,2,3,4,5,7$, and 11, in the vicinity of the crossing of the fracture line with the middle meningeal artery a dotted or a band-like extravasation shadow is visible along the middle meningeal artery. The case belonging to this pattern is most frequent (Photos 1,2 ). 
Pattern II : As shown in cases 9, 10, and 12 rather near the stem of the meningeal artery there exist two rows of shadow like a tram-track running parallel with its both sides proximal to its injuried site. This seems to result from a shunt developed between the middle meningeal artery and the vein (Photos 3, 4).

Pattern III : As shown in cases 8 and 9, a pseudoaneurysm is formed within a hematoma at the site of injury of the middle meningeal artery (Photos 5,6 ).

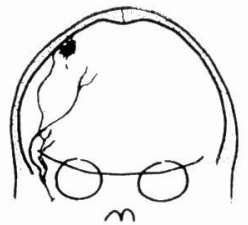

Case No.1 Pattern I

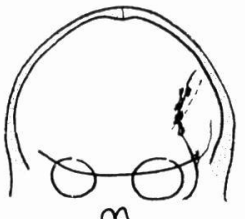

Case No. 2 Pattern I

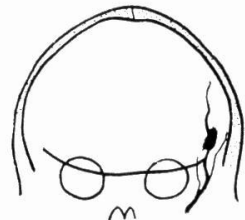

Case No. 3

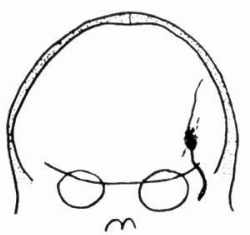

Case No. 4

Pattern I

Pattern
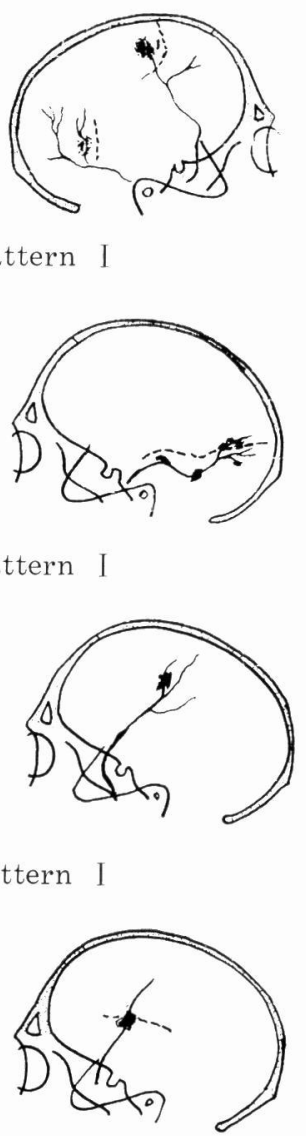

Fig. 1. Schema of the relation between the middle meningeal artery, fracture and extravasation shadow (Part 1 ). (A dotted line showed fracture.)
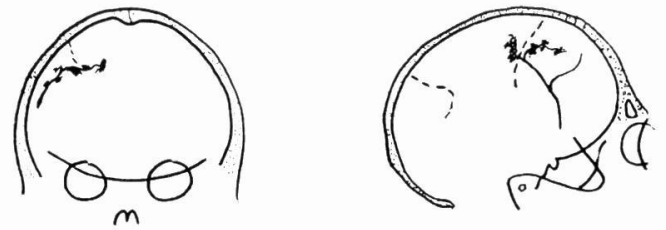

Case No. 5 Pattern I
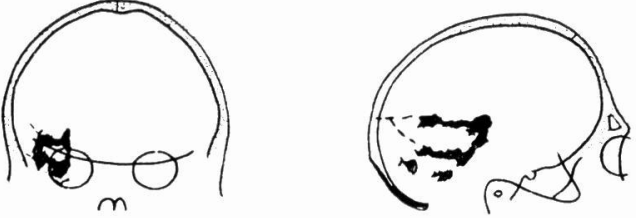

Case No.6 Pattern IV
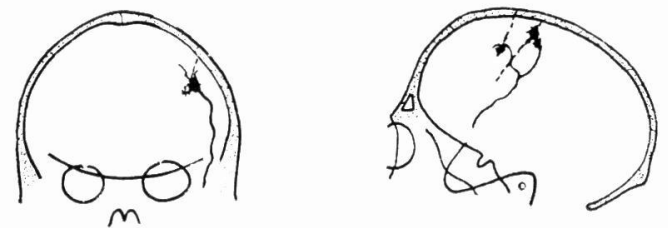

Case No. 7 Pattern I
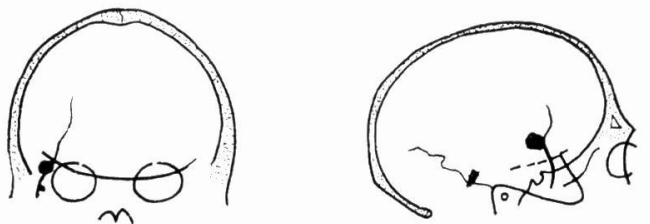

Case No. 8 Pattern III with I

Fig. 2. Schema of the relation between the middle meningeal artery, fracture and extravasation shadow (Part II). (A dotted line showed fracture.) 
Pattern IV : As seen in cases 6, a large quantity of contrast medium is visible in the fracture line as if it flowed out of the fracture line into the subgaleal area (Photos 7, 8).

Sometimes, 2 or more of the above patterns are observable together in cerebral angiographs of one and the same case.
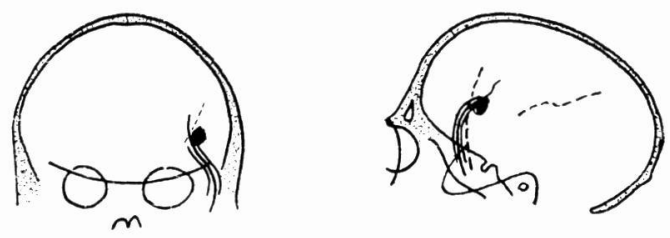

Case No. 9 Pattern II with III
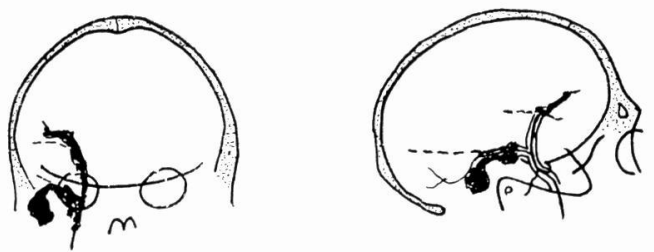

Case No. 10

Pattern II with I
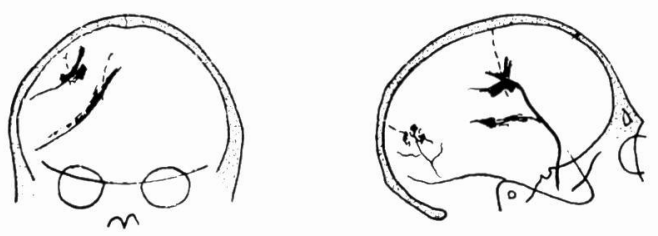

Case No.11 Pattern I
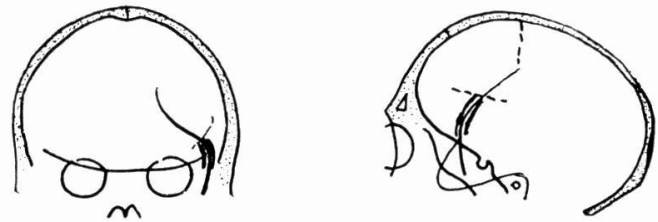

Case No. 12 Pattern II

Fig.3. Schema of the relation between the middle meningeal artery, fracture and extravasation shadow (Part III).

(A dotted line showed fracture.) 


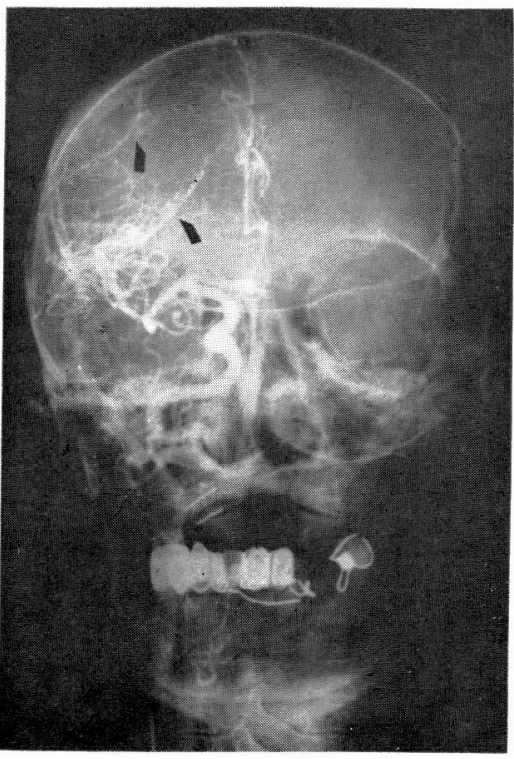

Photo. 1. Frontal view of Case No. 11. Anterior and posterior branches of the middle meningeal artery are expanded, along which a dotted extravasation shadow is visible (Pat. I).

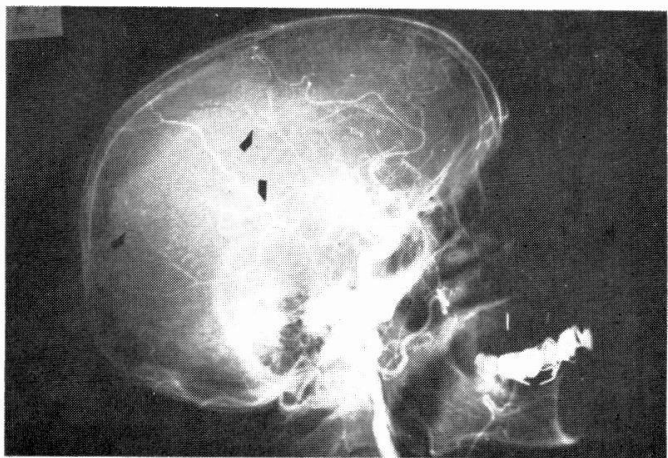

Photo. 2. Lateral view of Case No.11. Visible near the crossing of anterior and posterior branches of the middle meningeal artery with the fracture line is a dotted extravatation shadow (Pat. I).

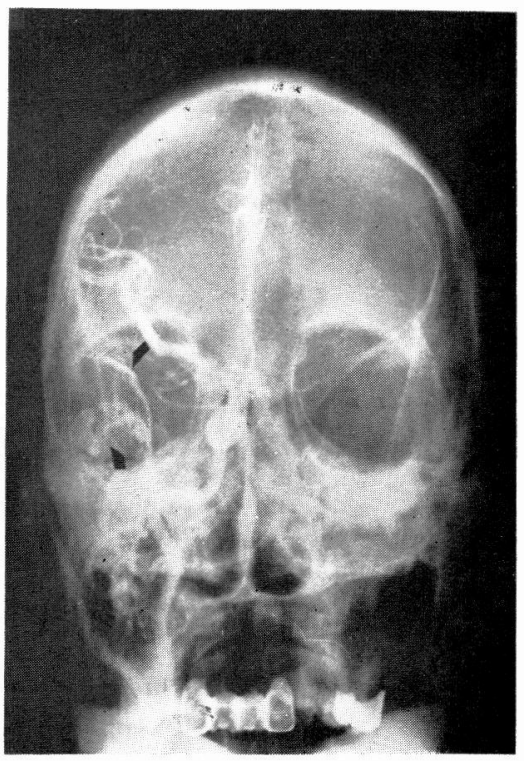

Photo. 3. Frontal view of Case No. 10. The middle meningeal artery is displaced inwards and along the anterior and posterior branches of it there is an extravasation shadow (Pat. II with pat. I ).

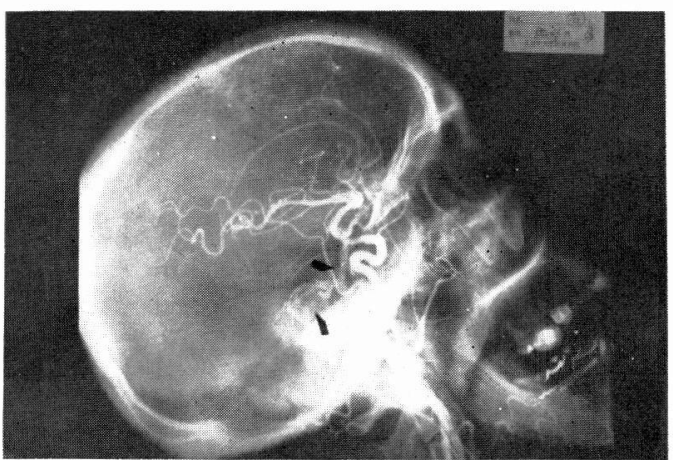

Photo.4. Lateral view of Case No. 10. In the crossing of the anterior and posterior branches of middle meningeal artery with the fracture line a tram-tracklike shadow is visible along its both sides together with a band-like one (Pat. II with pat. I ). 


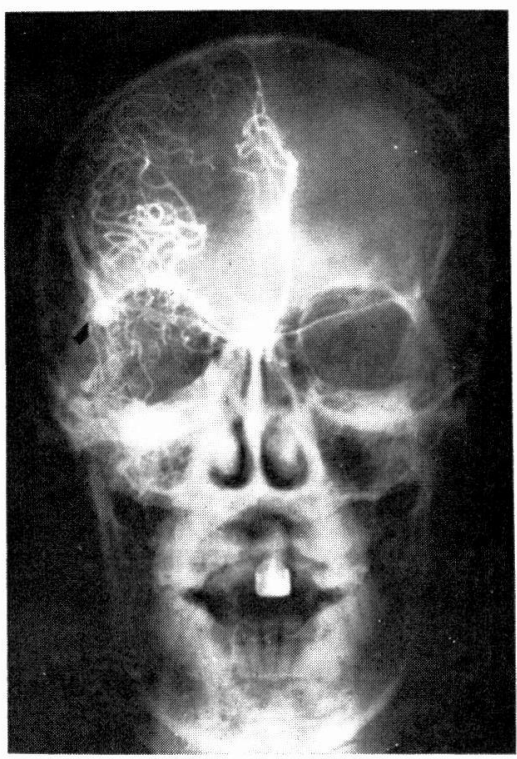

Photo.5. Frontal view of Case No. 8. The middle meningeal artery is displaced inwards, and on the anterior branch a pseudoaneurysm is visible and on the posterior branch an extravasation shadow (Pat. III with pat. I).

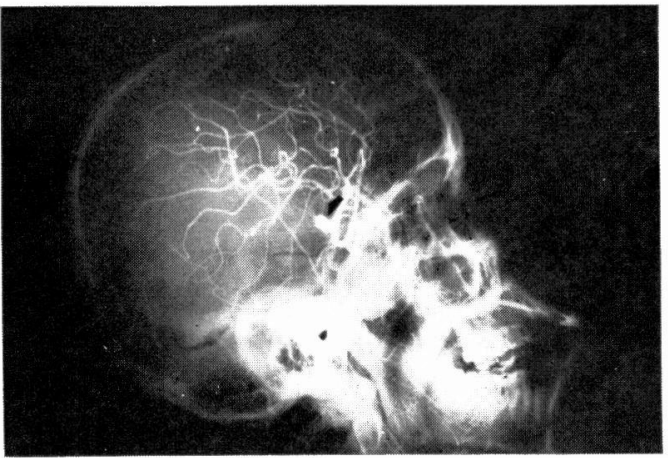

Photo. 6. Lateral view of Case No. 8. On the anterior branch of the middle meningeal artery above the fracture line is visible a pseudoaneurysm and on the posterior branch an extravasation shadow (Pat. III with pat. I).

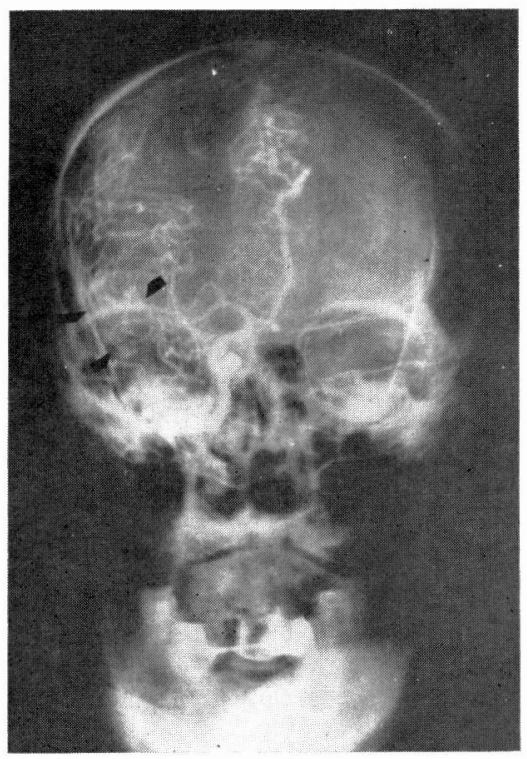

Photo.7. Frontal view of Case No. 6. Abundant contrast medium is seen filling the fracture lines (Pat. IV).

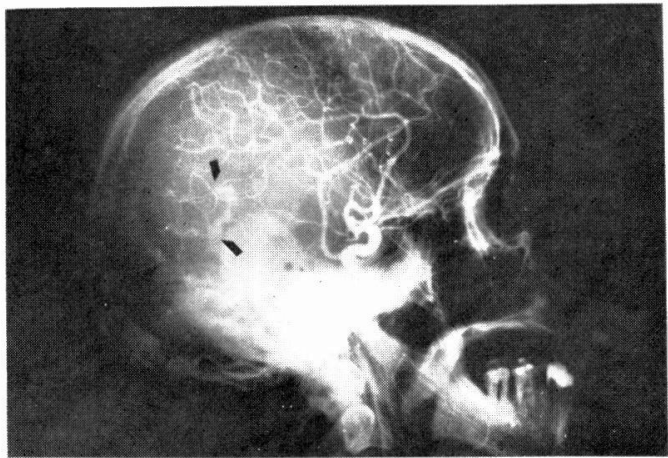

Photo. 8. Lateral view of Case No. 6. Abundant contrast medium is seen filling the fracture lines in the occipital region. It seems as if it flowed out of the fracture line in to the subgaleal area (Pat. IV). 


\section{DISCUSSION}

It was in the 1950's that cerebral angiography began to apply actively to the diagnosis of traumatic intracranial hematoma. From those days reports of authors who identified extravasation shadows in cerebral angiographs of extradural hematoma came to appear in succession. They were Jamieson (1952) ${ }^{1)}$, Lofstrom (1955) 2), Schulze (1957) 3), Vaughan (1959) 4), Huber(1962) 5), Leslie

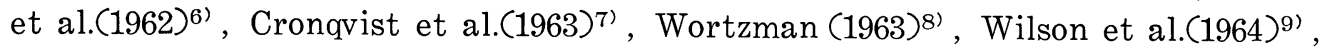
Paillas $(1964)^{10)}$, Schechter et al. $(1966)^{111}$, and others.

Among them, it was 7 cases of Huber ${ }^{5)}$ and 15 cases of Schechter et al. ${ }^{11}$, who most often reported such a case. In cerebral angiographs of 24 cases of extradural hematomas, Huber confirmed an extravasation shadow in 7 cases $(29.2$ $\%)$. We also observed it in 12 of 51 cases (23.5\%). From this, it may be assumed that the appearance of extravasation shadow in cerebral angiographs of extradural hematoma is not very rare.

Most of the bleeding sources of extradural hematoma lie in the injuried portion of the middle meningeal artery. The picture of extravasation of contrast medium from the tear of this artery varied in form, which therefore was divided into 4 groups depending on its patterns. Pattern I was most frequent, to which 6 of 12 cases examined belonged, presenting a diffusely extravasated picture of contrast medium along the above artery within an extradural hematoma presumably due to its tear in the fracture line. Pattern II showed a picture characteristic of a shunt formation between the middle meningeal artery and the vein, that is, a tram-track-like shadow along both sides of this artery. It was observed in 3 of 12 cases. Pattern III was one in which a pseudoaneurysm formed within a hematoma at the site of injury of the middle meningeal artery and was observed in 2 of 12 cases. Finally, pattern IV was observed only in one of 12 cases, in which abundant contrast material was found flowing into the fracture line. Angiographically, the above 4 patterns are thus distinguishable from each other, and 2 or more patterns sometimes appear together in cerebral angiographs of one and the same case. Schechter et al. ${ }^{11}$ also divided these angiographic extravasation shadows into 4 groups nearly corresponding to our classification.

Huber ${ }^{5)}$ stated that the shorter the interval from injury to cerebral angiography was, the more frequently the extravasation shadow appeared. Out of 7 cases in which he confirmed an extravasation shadow, in 5 cases it appeared within 6 hours and in the other 2 within 32 hours. In our 12 cases, it appeared within 27 hours in 9 cases and in 3 to 8 days in the remainder 3 . Out of 15 cases of Schecter et al. ${ }^{11)}$, in 5 cases it also showed itself in 3 to 8 days. From this, it may be concluded that in the case of performing cerebral angiography at a short interval from injury an extravasation shadow is often to be identified, but that even after a considerably long time elapsed after injury there is a fair possibility of demonstrating it. 
The extravasation shadow seen in cerebral angiographs of traumatic intracranial hematomas is mostly confined to the case of extradural hematomas. This fact seems to be very helpful for discriminating intradural hematomas from extradural ones together with the finding that the middle meningeal artery is displaced inwards from the inner table of the skull.

\section{CONCLUSION}

Out of 51 cases of cerebral angiography of traumatic extradural hematomas, in 12 cases an extravasation shadow of contrast medium from the injuried portion of the middle meningeal artery was observed. The extravasation shadow in these 12 cases was divided into 4 types depending on its pattern.

Since the shadow is very specific for extradural hematomas, it may be therapeutically of much use for the differentiation between intra- and extradural hematomas by cerebral angiography.

\section{REFERENCES}

1) Jamieson, K. G. : Unusual case of extradural hematoma. Radiology, 65, 847-856, 1951.

2 ) Lofstrom, J. E., Webster, J. E., and Gurdjian, E. S. : Angiography in evaluation of intracranial trauma. Radiology, 65, 847-856, 1955.

3) Schulze, A. : Seltene Verlaufsformen epiduraler Hämatome. Zentralbl. Neurochir. 17, 40-47, 1957.

4) Vaughan, B. F. : Middle meningeal hemorrhage demonstrated angiographically. Brit. J. Radiol., 32, 493-494, 1959.

5) Von Huber, P. : Die Verletzungen der Meningeal Gefässe beim Epiduralhämatom in Angiogramm. Fortschr. Roentgenstr., 96, 207-220, 1962.

6) Leslie, E. V., Smith, B. H. and Zoll, J. G. : Value of angiography in head trauma. Radiology, 78, 930-940, 1962.

7 ) CRONQVist, S. and Kobler, R. : Angiography in epidural hematomas. Acta radiol, 1, 42-52, 1963.

8) Wortzman, G. : Roentgenologic aspects of extradural hematoma. Amer. J. Roentgenol., 90, 462-471, 1963.

9) Wilson, C. B. and Cronic, F. : Traumatic arteriovenous fistulas involving the middle meningeal vessels. J. A. M. A. 188, 953-957, 1964.

10) Paillas, J. E., Bonnal, J. and Lavieille, J. : Angiographic image of false aneurysmal sac caused by rupture of median meningeal artery in the course of traumatic extradural hematoma. Report of three cases. J. Neurosurg. 21, 667-671, 1964.

11) Schechter, M. M., Zingesser, L. H. and RAyport, M. : Torn meningeal vessels : An evaluation of a clinical spectrum through the use of angiography. Radiol., 86, 686$695,1966$. 